Reprod. Nutr. Dévelop., 1987, 27 (1 B), 305-306.

\title{
Influence de la photopériode sur l'évolution du poids vif et l'activité ovarienne post partum chez les vaches allaitantes
}

\author{
J. P. GAREL, D. GAUTHIER, M. PETIT, J. THIMONIER $\left({ }^{*}\right)$
}

Laboratoire de l'Elevage Bovin, I.N.R.A., Theix, 63122 Ceyrat.

(*) Physiologie de la Reproduction, I.N.R.A., Nouzilly, 37380 Monnaie.

Summary. The effect of increasing the duration and/or the intensity of light was studied in suckled cows ; group 1 received 300 lux for $16 \mathrm{~h}$ and group 2 was given 300 lux for the whole day. The postpartum anovulatory period decreased in both groups compared to control group 3. Thus, 60,45 and $20 \%$ (groups 1, 2 and 3, respectively) of the cows showed ovarian activity at 70 postpartum days.

En France, la plupart des vaches allaitantes mettent bas pendant l'hiver et, lorsqu'elles sont maintenues à l'attache, sont souvent gardées dans des stabulations très sombres. Ce mode de stabulation, la saison de mise-bas, le niveau alimentaire insuffisant, pourraient être en partie responsables du faible pourcentage de femelles allaitantes cycliques au moment souhaité pour la mise à la reproduction (Terqui et al., 1981). Or, des " jours longs " favorisent l'activité ovarienne (Hansen, 1985). Le rôle de la photopériode sur la reprise de l'activité ovarienne pendant la période post partum a donc été étudié chez les vaches Salers allaitantes.

Matériel et méthodes. Soixante vaches de race Salers vêlant entre le 29/12 et le 28/02 sont placées du 15 novembre au 2 avril dans une stabulation entravée sombre (25 lux en moyenne) semblable à de nombreuses étables traditionnelles de troupeaux bovins allaitants. A partir du 12 décembre, elles sont divisées en 3 lots de 20 vaches chacun, comparables quant aux poids, date et rang de vêlage. Le lot 1 reçoit alors, $16 \mathrm{~h}$ par jour, une lumière de 300 lux émise par des tubes fluorescents fixés au-dessus de la tête des animaux. Le lot 2 reçoit la même intensité lumineuse, pendant une durée égale à la durée légale du jour (Météorologie Nationale). Le lot 3 , lot témoin, ne reçoit de la lumière supplémentaire que pendant la durée des interventions du personnel dans l'étable. Cette lumière est toutefois peu perceptible au niveau des yeux des animaux. Tout au long de l'expérience la ration alimentaire, composée de $85 \%$ de foin et $15 \%$ d'orge, est limitée et modulée en fonction du poids et du stade de gestation ou de la production laitière, de façon à ce que le niveau énergétique soit inférieur de 1 UFL aux recommandations d'alimentation des vaches allaitantes. La quantité distribuée est contrôlée tous les jours et les refus cumulés sur une semaine. Les vaches sont pesées tous les 14 jours et dans les 4 jours suivant le vêlage. Elles allaitent un seul veau 2 fois par jour. La production laitière est mesurée toutes les deux semaines par pesée du veau avant et après tétée (Le Neindre, 1973). Les veaux sont logés dans un local séparé des mères et ne reçoivent aucun traitement photopériodique. Un prélèvement sanguin est effectué une fois par semaine sur chacune des vaches à partir du $30^{\mathrm{e}}$ jour post partum afin de détecter les ovulations par l'étude des variations de la progestéronémie (Thimonier, 1978).

Résultats et discussion. La quantité de matière sèche consommée ne diffère pas entre les lots et est en moyenne de $7,1 \pm 0,5 \mathrm{~kg} / \mathrm{j}$ pendant le $8^{\mathrm{e}}$ mois de gestation, $8,1 \pm 0,5 \mathrm{~kg} / \mathrm{j}$ pendant le $9 \mathrm{e}$ mois et de $10,5 \pm 0,9 \mathrm{~kg} / \mathrm{j}$ pendant la lactation ; ceci constitue une ration apportant une quantité d'énergie inférieure de 15 à $20 \%$ aux recommandations alimentaires pour des vaches allaitantes. 
Le poids des veaux à la naissance est identique pour les deux lots $(40,4 \pm 4,0 \mathrm{~kg})$. Il en est de même de la production laitière des mères au cours des 45 premiers jours post partum $(9,2 \pm 2,1 \mathrm{~kg} / \mathrm{jour})$. Le poids vif moyen des animaux (tabl. 1) ne diffère pas entre les lots au cours de l'expérience. En revanche, la perte de poids entre -40 et +40 jours post partum est plus importante pour les femelles du lot témoin $(72 \mathrm{~kg})$ que pour les autres femelles $159 \mathrm{~kg}$; $P<0,05)$. L'augmentation de la durée ou de l'intensité de la lumière entraînerait donc une meilleure utilisation de la ration au profit d'une moindre perte de réserves corporelles et non d'une production laitière accrue comme cela a été montré chez la brebis traite (Bocquier, 1985). II s'agit toutefois dans notre expérience de femelles allaitantes pour lesquelles le veau constitue le principal facteur limitant la production laitière au début de la lactation.

TABL. 1. - Poids vif, production laitière et activité ovarienne post partum de vaches Salers recevant du 12 décembre au 2 avril: Lot $1: 300$ Lux pendant 16 h consécutives; Lot $2: 300$ lux pendant la durée légale du jour; Lot 3 : aucune lumière supplémentaire.

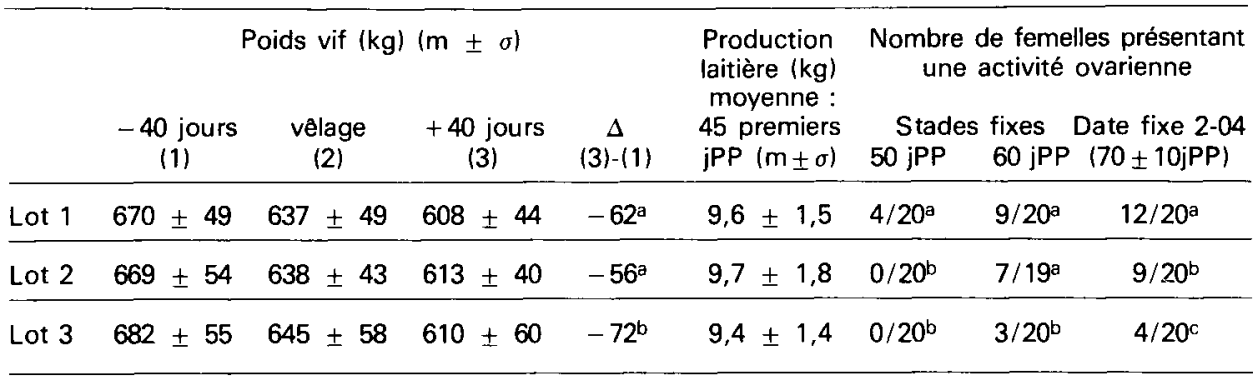

Pour un même paramètre les valeurs avec des lettres identiques ne sont pas significativement différentes (jPP = jours post partum).

A 50 jours post partum, le pourcentage de femelles cycliques de $20 \%$ pour le lot 1 , est nul pour les 2 autres lots $(P<0,05)$. Dix jours plus tard, ces pourcentages sont de 45,37 et 15 pour les lots 1,2 et $3(P<0,05 ;$ tabl. 1 ). A la date du 2 avril (stade post partum moyen de $70 \pm 10$ jours) ces pourcentages sont alors respectivement de 60, 45 et $20(P<0,01)$ pour l'ensemble des 20 vaches de chaque lot (tabl. 1).

Cet effet de la photopériode sur la cyclicité des vaches Salers élevées en étable traditionnelle est identique à celui décrit par Hansen (1985) pour les génisses laitières, et montre que la lumière peut constituer un des facteurs limitant la cyclicité des bovins allaitants. Bien que les mécanismes physiologiques en cause soient encore inconnus, il est probable que cette action passe, comme chez les ovins, par une action au niveau du système nerveux central (Ortavant et al., 1985).

Une photopériode claire longue et/ou intense favorise donc la reprise de l'activité ovarienne post partum chez les vaches allaitantes maintenues en stabulation entravée et sous alimentées pendant la période hivernale. De plus, elle semble améliorer l'utilisation de la ration en réduisant la perte de poids.

Bocquier F., 1985. Thèse de Docteur-Ingénieur, I.N.A.P.G., Juin 1985.

Hansen P. J., 1985. Livest. Prod. Sci., 12, 309-327.

Le Neindre P., 1973. Ann. Zootech., 22, 413-422.

Ortavant R., Pelletier J., Ravault J. P., Thimonier J., Volland-Nail P., 1985. Oxford Rev. reprod. Biol., 7, 305-345.

Terqui M., Chupin D., Gauthier D., Pérez N., Pélot J., Mauléon P., 1981. Seminar C.E.E., Sept. 30-

Oct. 2, 1981, Munich, ed. Karg and Schallenberger, pp. 384-408.

Thimonier J., 1978. Ann. Méd. vét., 122, 81-92. 